\title{
A network of ontology networks for building e-employment advanced systems ${ }^{\text {is }}$
}

\author{
Boris Villazón-Terrazas, Jaime Ramírez*, Mari Carmen Suárez-Figueroa, Asunción Gómez-Pérez \\ Ontology Engineering Group, Departamento de Inteligencia Artificial, Facultad de Informática, Universidad Politécnica de Madrid, Spain
}

\section{A R T I C L E I N F O}

\section{Keywords:}

Ontological engineering

Human resources management

Semantic interoperability

\begin{abstract}
A B S T R A C T
This paper presents the development of a network of ontology networks that enables data mediation between the Employment Services (ESs) participating in a semantic interoperability platform for the exchange of Curricula Vitae (CVs) and job offers in different languages. Such network is formed by (1) a set of local ontology networks that are language dependent, in which each network represents the local and particular view that each ES has of the employment market; and (2) a reference ontology network developed in English that represents a standardized and agreed upon terminology of the European employment market. In this network each local ontology network is aligned with the reference ontology network so that search queries, CVs, and job offers can be mediated through these alignments from any ES. The development of the ontologies has followed the methodological guidelines issued by the NeOn Methodology and is focused mainly on scenarios that involve reusing and re-engineering knowledge resources already agreed upon by employment experts and standardization bodies. This paper explains how these methodological guidelines have been applied for building e-employment ontologies. In addition, it shows that the approach to building ontologies by reusing and re-engineering agreed upon non-ontological resources speeds the ontology development, reduces development costs, and retrieves knowledge already agreed upon by a community of people in a more formal representation.
\end{abstract}

(c) 2011 Elsevier Ltd. All rights reserved.

\section{Introduction}

Human resources managers have discovered that the Web can be a very effective communication vehicle. Although most businesses rely on diverse recruiting channels such as newspaper advertisements, online job exchange services, trade fairs, co-worker recommendations and human resources advisors, online personnel marketing is increasingly used because of their cost cutting results and efficacy. Public and private Employment Services (ES) are becoming more and more important for Public Administrations since their social implications on sustainability, workforce mobility and equal opportunities have a fundamental and strategic importance to both central and local Governments. Workforce mobility and political decisions in a Pan-European scope require wide European consortia to share and exchange employment information (mainly job offers and CVs written in different languages). This eemployment information system should involve different ESs that, in turn, manage their own repositories where sensitive and crucial data are recorded to support day-to-day operations. Moreover, the knowledge behind these data is implicitly encoded by means of ES specific models. However, the need to exchange and reconcile

\footnotetext{
Work funded by SEEMP (FP6-027347) and NeOn (FP6-027595) EU Projects.

* Corresponding author.

E-mail addresses: bvillazon@fi.upm.es (B. Villazón-Terrazas), jramirez@fi.upm.es (J. Ramírez), mcsuarez@fi.upm.es (M.C. Suárez-Figueroa), asun@fi.upm.es (A. Gómez-Pérez).
}

these data, possibly written in different languages, among the different ESs requires us to make explicit the ES specific models. Ontologies play an important role for that purpose since they are used to give full detail of the meaning of the information contained in many e-government and e-employment applications.

Various approaches have been designed to deal with the semantic integration of heterogeneous information sources by means of ontologies, either in data sources or in mediators (Wache et al., 2001). In this paper we present the approach adopted in the SEEMP $^{1}$ (Single European Employment MarketPlace) project, where a network of ontologies provides the semantics enabling the data mediation. An ontology network or a network of ontologies is defined, according to Haase et al. (2006), as a collection of ontologies (called networked ontologies) related together through different meta-relationships such as mapping, modularization, version and dependency relationships.

The network of ontology networks presented in this paper comprises two kind of components: (1) local ontology networks, one for each ES, that are language dependent and make explicit the knowledge implicitly encoded in each ES specific model; and (2) a reference ontology network developed in English that mediates among the local ontology networks and unifies the terminology and conceptualization of the domain. Thus, the SEEMP project relies on a network of ontology networks because both the reference ontology

\footnotetext{
1 http://www.seemp.org.
} 
and the local ontology are ontology networks by themselves. Furthermore, the whole e-employment ontology network is the result of putting together the reference ontology network and the local ontology networks, and defining a set of alignments or semantic mappings between each local ontology network and the reference ontology network.

In the last few years, and within the context of the NeOn project $^{2}$ a scenario-based methodology, called the NeOn Methodology, has been developed. This methodology aims to build ontology networks by reusing and re-engineering knowledge resources. The development of the SEEMP ontologies has required several reverse engineering processes that take advantage of the existence of nonontological resources already agreed on either in each local ES or in the European scope in the form of standards. The fact that a consensus in the terminology defined by a non-ontological resource has been reached must not be underestimated since this consensus helps to reduce development costs drastically. The NeOn Methodology (Suárez-Figueroa, 2010) provides detailed methodological guidelines for the different processes and activities involved in the ontology network development process.

This paper shows how the NeOn Methodology has been applied successfully in the development of the SEEMP ontology networks. More concretely, the SEEMP prototype has required the development of a reference ontology network and two local ontology networks (for Telmi ES and EURES ES, respectively). In addition, in this paper we have tried to demonstrate that the approach to building ontologies by reusing and re-engineering agreed upon nonontological resources allows building ontologies faster and with less resources. Moreover, another advantage of this approach is that the ontologies built in this way can reach an immediate consensus.

The remaining of the paper is organized as follows: Section 2 presents the goals of the SEEMP project and outlines the proposed interoperability architecture. Section 3 summarizes the NeOn Methodology and emphasizes those aspects most relevant for the development of the SEEMP ontology networks. Section 4 shows how the NeOn guidelines have been applied in building the SEEMP ontologies. Section 5 presents our conclusions and the final section focuses on new lines of research derived from this work.

\section{Related work}

In this section we present the most outstanding research efforts for applying ontologies to the human resources domain. Gualtieri and Ruffolo (2005) and Terziev et al. (2005) present ontologies that represent organizational knowledge of a business. The former propose an ontology-based framework organized as a two-level family of ontologies: the first-level ontologies represent organizational background, whereas the second-level ontologies formally represent human resources, business processes, technical resources and knowledge objects. The latter, on the other hand, present a modular ontology, PROTON, that was built by reusing the KIMO ontology ${ }^{3}$ for modelling people, organizations, locations, numbers, dates and addresses.

There are also interesting contributions in the area of the competency management. Thus, for example, Trichet and LeclFre (2003) introduces CommOn (Competency Management through Ontologies), a generic framework dedicated to the development of Competency Based Systems. Their framework is based on the following concepts: competency, resources, context, and objective. Four types of resources are covered in their work: theoretical knowledge, procedural knowledge, skills and behavioral aptitudes. Biesalski and Abecker (2005) demonstrate how the use of competency catalogues (as lightweight ontologies) helps to

\footnotetext{
${ }^{2}$ http://www.neon-project.org.

3 http://www.ontotext.com/kim.
}

integrate process in human resources and present a german competency catalog containing information about competency and training. To show this they reused a catalogue stored in a simple flat database.

The works of Bizer et al. (2005) and Mochol and Paslaru (2006) are specially remarkable in the way they reuse and integrate widespread standards and classifications when creating an ontology for e-employment in a national scope (Germany). Two examples of these standards and classifications are: SOC, Occupation Code (Berufskennziffer - BKZ), ${ }^{4}$ Classification of Industry Sector (WZ2003), ${ }^{5}$ etc. Their ontology comprises the following subontologies: Education, JobPositionSeeker, Industry, JobPositionPosting, Organization, Skills and Person. However, apart from mentioning the standards employed in each of these subontologies, they do not give further details about the composition of these subontologies.

Another work related to e-employment that deals with the reusing of standards and classifications is Dorn, Naz, and Pichlmair (2007), which shows the development of an ontology modelling job descriptions, competences and learning objects for two different projects: a meta-search engine for searching for jobs in job portals and an university competence management system. In the building of this ontology Standard Occupational System (SOC) ${ }^{6}$ and HR-XML recommendation were employed.

Another good example of e-employment ontology is GarcíaSánchez, Martínez-Béjar, Contreras, Fernández-Breis, and Castellanos-Nieves (2006) where the ontology supports a search engine that provides intelligent matches between job offers and candidates curricula in a job portal bounded to a rural area. Nevertheless, the development of this ontology does not rely on any widespread standard or classification.

So far, any previous e-employment ontology has been built as a network of ontology networks, or has been designed to support interoperability of job offers and CVs in an European scope. Therefore, to support such type of interoperability we built a reference ontology, which required a greater effort than that employed for building an ontology intended for a local scope. Specifically, this effort was significantly greater when selecting and adopting international standards and classifications. Additionally, in order to support a suitable mediation among local ontologies, our reference ontology was created to provide a richer vocabulary than previous ontologies bounded solely to a regional or national scope.

Concerning methodological issues, none of the papers mentioned above except for Mochol and Paslaru (2006) documents the use of any methodology for reusing ontological or non-ontological resources. In this regard, it should be noted that the reusing of non-ontological resources was a key aspect in the development of our reference ontology, because, as we will comment in Section 5.1.4, all the standards and classifications that we adopted are considered non-ontological resources.

\section{A semantic interoperability platform for e-employment}

The main use case scenario consists of a job seeker that submits his/her search criteria through a Web site of a local ES. These search criteria describe the characteristics of the job offer (JO) in which the job seeker is interested (type of occupation, contract type, location of the job, compensation conditions, etc.). Normally, after this submission, the job seeker obtains some JOs from the local database of the ES. However, if the job seeker wishes to know about other JOs, which may be stored in foreign ESs, he would have to submit his/her search criteria in the Web site of other ESs, probably in a language other than his/her own. For this scenario, SEEMP

\footnotetext{
${ }^{4}$ http://www.arbeitsamt.de/hst/markt/news/BKZ_alpha.txt.

5 http://www.destatis.de/allg/d/klassif/wz2003.htm.

${ }^{6}$ http://www.bls.gov/soc/.
} 


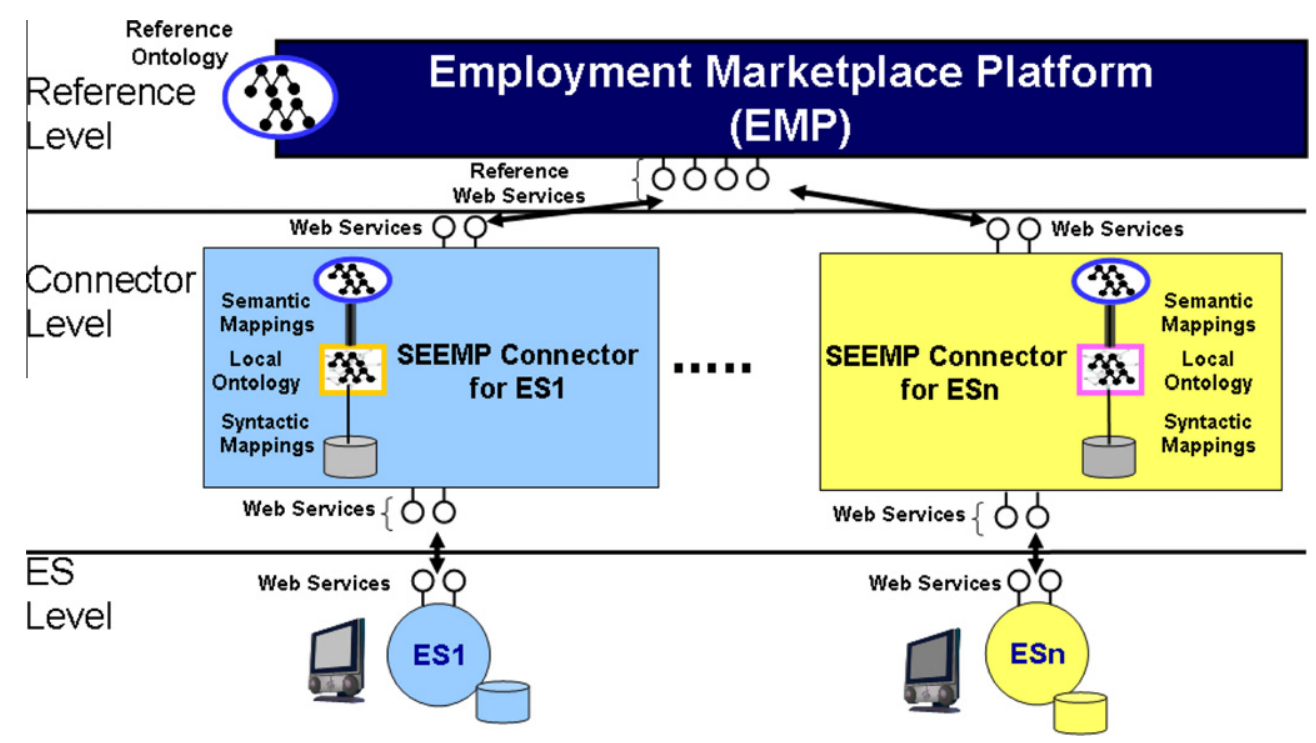

Fig. 1. SEEMP interoperability platform.

proposes a technical solution in which the job seeker would only have to submit his/her search criteria once since the ES will forward his/her search query to other ESs through a semantic operability platform, and then the job seeker will be able to retrieve JOs from different ESs, possibly located in different countries and written in a different language. In addition, the JOs found will be provided to the job seeker in his/her own language and in terms of occupations, education levels, etc. used by his/her ES. Likewise, SEEMP covers also a similar use case scenario for searching CVs.

This scenario poses at least two technical challenges because service and data heterogeneity issues must be solved when mediating the search query and the JOs compliant with this search query. The problem of service heterogeneity arises because each ES may expose their Web Services through a different Web Services interface; the problem of data heterogeneity, on the other hand, must be tackled at three levels: at the lexical level, because the mediated search queries, CVs, and JOs can be written in different languages. Hence, the solution must support a multilingual scenario; at the syntactic level, because ESs use different XML schemas for exchanging CVs, JOs and search queries through Web Services; and at the conceptual level, since the knowledge used by each ES is implicitly encoded with ES specific models. That means that each ES, possibly belonging to a different country, may use different codes or classifications, which are close-related to its own local reality, for languages, occupations, education levels, skills, etc.

Thus, in order to overcome these heterogeneity problems, the interoperability platform proposed is structured in the following three logic levels (Valle et al., 2008) (see Fig. 1):

- The Reference level, which comprises the Employment Marketplace Platform, that is, the core component of the architecture, and its goals are (1) to broadcast a search query, from one of the ES to the rest of ESs; (2) to collect the results provided by each ES and rank them; and (3) to provide these results to the ES submitting the query. At this level all the information handled, such as queries, CVs, and JOs, is expressed in terms of a reference ontology network.

- The Connector level, which contains a connector component for each ES. This connector is in charge of coping with the heterogeneity issues posed by each ES, which implies, among other things, translating the queries, CVs, and JOs expressed in terms of ES local data sources into ontological instances expressed in terms of the reference ontology network, and vice versa.
- The ES level, which includes the ESs connected to the marketplace through the SEEMP Connectors.

The SEEMP project proposed three different architectures for the connector component, though the two-step semantic XML translation was the recommended option (see Valle et al., 2008 for further details), since this translation offers the cleanest and most maintainable solution. According to this approach, the mediation process is carried out in two steps, one syntactic and one semantic. This means that for lifting a CV from the ES to the Employment Marketplace Platform (EMP), it is necessary to perform a syntactic transformation in which the XML representation of the CV is translated into instances in terms of a local ontology network; then, in the semantic step these instances of the local ontology network are transformed into instances of the reference ontology network. Likewise, in order to lower a CV from the EMP to the ES, the same steps are applied but in the opposite order. Whereas to perform the syntactic transformation the $\mathrm{XSLT}^{7}$ technology is applied, to perform the semantic step a WSMX ${ }^{8}$ Data Mediation component is put in action.

As a result of adopting this architecture for all the connectors (see Fig. 1), we could say that the interoperability platform is based on a network of ontology networks formed by a reference ontology network that represents a standardized and agreed upon view of the employment market at the European level; and a set of local ontology networks, each of them representing the local and particular view that each ES has of the employment market. Furthermore, the reference ontology network is aligned with each local ontology network, so that the aforementioned semantic step is possible. In Section 5 will be shown that each of these ontologies constitutes an ontology network by itself.

\section{NeOn Methodology for building ontology networks}

The NeOn Methodology ${ }^{9}$ is a scenario based methodology that provides guidance for all key aspects of the ontology engineering process, namely, collaborative ontology development, reuse of onto-

\footnotetext{
Extensible Stylesheet Language http://www.w3.org/TR/xslt.

${ }^{8}$ Web Service Modelling eXecution environment, http://www.wsmx.org/.

${ }^{9}$ A book about this methodology is going to be published before the end of 2011. In the meantime a summary can be found at http://www.neon-project.org/nw/ NeOn_Book.
} 


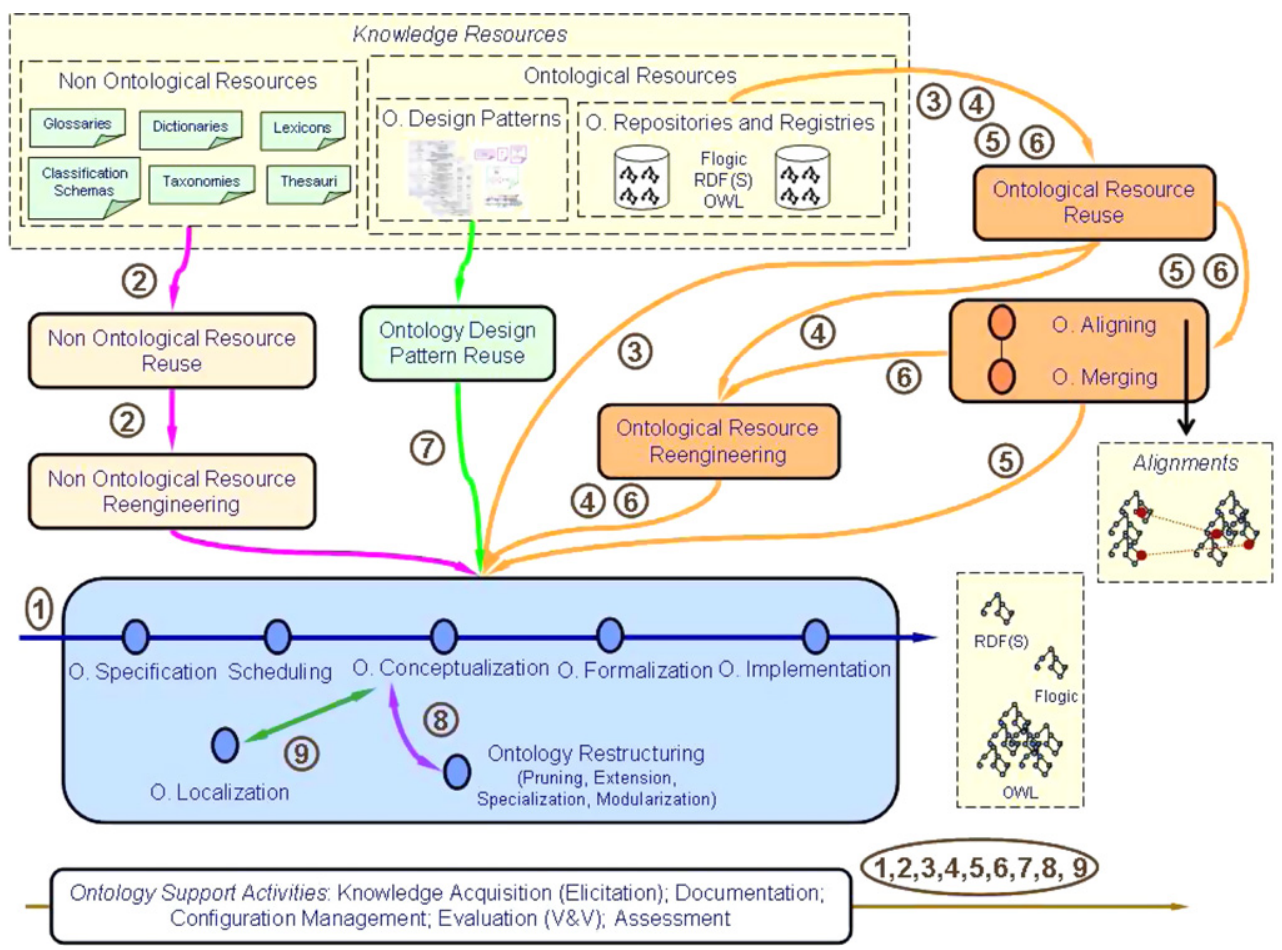

Fig. 2. Scenarios for building ontologies and ontology networks.

logical and non-ontological resources, and the evolution and maintenance of ontology networks.

In contrast to other approaches that provide methodological guidance for ontology engineering, the NeOn Methodology does not prescribe a rigid workflow, but instead it suggests pathways as well as processes and activities for a variety of scenarios. The nine scenarios identified in the NeOn Methodology cover commonly occurring situations, e.g., when available ontologies need to be re-engineered, aligned, modularised, localized (to support different languages and cultures) and integrated with non-ontological resources, such as folksonomies or thesauri. These scenarios are for building collaboratively ontologies and ontology networks, and special emphasis is placed on reusing and re-engineering knowledge resources (ontological and non-ontological).

Fig. 2 presents the set of the nine most plausible scenarios for building ontologies and ontology networks. The directed arrows with associated numbered circles represent the different scenarios. Each scenario is decomposed into different processes or activities. Processes and activities are represented with coloured circles or with rounded boxes and are defined in the NeOn Glossary of Processes and Activities (Suárez-Figueroa, 2010; SuárezFigueroa \& Gómez-Pérez, 2008). Fig. 2 also shows (as dotted boxes) the existing knowledge resources to be reused, and the possible outputs that result from the execution of some of the presented scenarios.

Next, we provide a list of the most common scenarios that may unfold during the ontology network development, though the list cannot be considered exhaustive. For a detailed explanation of the scenarios, please refer to Suárez-Figueroa (2010).

- Scenario 1: from specification to implementation. The ontology network is developed from scratch (without reusing available resources). Developers should specify ontology requirements. After that, it is advisory to carry out a search for potential resources to be reused. Then, developers should perform the scheduling activity and carry on with the plan.
- Scenario 2: reusing and re-engineering non-ontological resources (NORs). Developers should carry out the NOR reuse process for deciding, according to the ontology requirements, which NORs can be reused to build the ontology network. Then, they should re-engineer the selected NOR into ontologies. Guidelines for this scenario can be found in Villazón-Terrazas, Suárez-Figueroa, and Gómez-Pérez (2010), Villazón-Terrazas (2011).

- Scenario 3: reusing ontological resources. Developers use ontological resources (ontologies as a whole, ontology modules, and/or ontology statements) to build ontology networks. Guidelines for applying this scenario can be found in Suárez-Figueroa (2010).

- Scenario 4: reusing and re-engineering ontological resources. This scenario unfolds in those cases in which the outcomes of Scenario 3 are not exactly useful as they are and the ontological resources should be re-engineered to serve to the intended purpose.

- Scenario 5: reusing and merging ontological resources. This scenario unfolds only in those cases in which several ontological resources within the same domain are selected for reuse and when ontology developers wish to create a new ontological resource from two or more ontological resources.

- Scenario 6: reusing, merging and re-engineering ontological resources. Ontology developers reuse, merge, and re-engineer ontological resources in the ontology network building. This scenario is similar to Scenario 5; however, here developers decide not to use the set of merged resources such as they are but to re-engineer them.

- Scenario 7: reusing ontology design patterns (ODPS). Ontology developers access repositories to reuse ODPs. Guidelines for performing this scenario can be found in Presutti, Daga, Gangemi, and Blomqvist (2009).

- Scenario 8: restructuring ontological resources. Ontology developers restructure (by modularizing, pruning, extending, and/or specializing) ontological resources to be integrated in the ontology network.

- Scenario 9: localizing ontological resources. Ontology developers adapt an ontology to other languages and culture communities, 


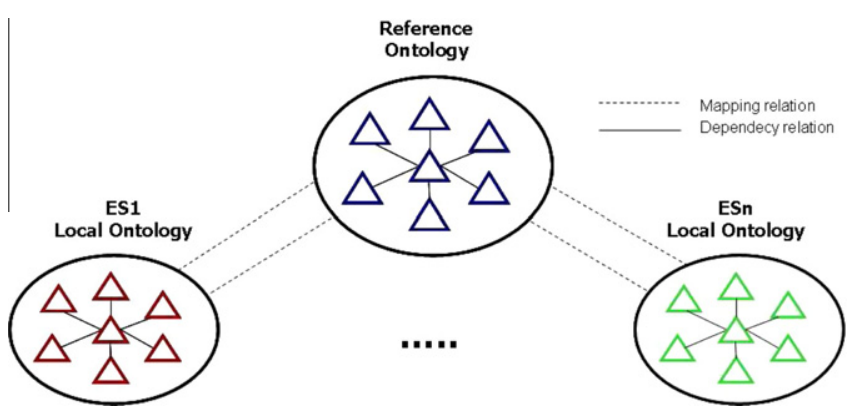

Fig. 3. SEEMP network of ontology networks.

thus obtaining a multilingual ontology. Guidelines to localize ontological resources can be found in Espinoza, MontielPonsoda, and Gómez-Pérez (2009).

Knowledge acquisition, documentation, configuration management, evaluation and assessment should be carried out all along the ontology development (during the whole ontology network development), that is, in any scenario used for developing the ontology network.

It is worth mentioning that these scenarios can be combined in different and flexible ways, and that any combination of scenarios should include Scenario 1 because this scenario is made up of the core activities that have to be performed in any ontology development. Indeed, as Fig. 2 shows, the results of any other scenario should be integrated in the corresponding activity of Scenario 1.

\section{5. e-Employment ontology network development}

This section aims to show how we built the e-employment ontology network by using Scenarios 1, 2, 4 and 5 of the NeOn Methodology. First we explain the reference ontology (RO) network development process, and then the development of the local ontology networks, one for each ES. Finally, we briefly outline how these networks of ontologies are integrated by means of Scenario 5 to obtain the network of ontology networks. The result of integrating the local ontologies networks and the RO network is depicted in Fig. 3. As can be seen in the figure, the solution proposed is a network of ontology networks in which both the RO network and the local ontology networks are ontology networks by themselves and are connected through the definition of a set of alignments.

\subsection{Reference ontology network development}

The RO network ${ }^{10}$ acts as a common "language" because it provides a rich set of terms to describe the details of a JO and the CV of a job seeker, as well as the details of a search query for CVs or JOs. In the next paragraphs we will focus on the modelling of the CVs and JOs, and we will skip the modelling of the search queries. It should be mentioned that when we started the project and with the purpose of reusing human resources management ontologies, we considered some previous works on this area (Biesalski \& Abecker, 2005; Bizer et al., 2005; Gualtieri \& Ruffolo, 2005; Mochol \& Paslaru, 2006; Terziev, Kiryakov, \& Manov, 2005; Trichet \& LeclFre, 2003). Nevertheless, the ontologies reported in these works either are not publicly available, or are their scope limited to a certain country and not to the whole Europe. Hence, these ontologies could not be reused in the development of the RO.

For building the RO, we followed the activities specified in Scenario 1 , therefore we began with the ontology requirement specification.

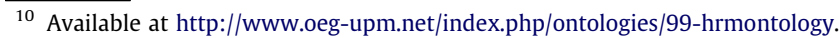

\subsubsection{Reference ontology network requirements specification}

As a result of this activity we produced the ontology requirements specification document (ORSD) (SEEMPD32, 2006), which includes information (see Fig. 4) about the purpose, the scope, the level of formality, the intended users, the intended uses of the RO, a set of 60 competency questions ${ }^{11}$ and a pre-glossary of terms. The competency questions were grouped into the following areas: job seeker information, job offer information, compensation (salary and bonus), driving license, economic activity, education (level and knowledge field), geography, labour regulatory (contracts and work time), language (languages and expertise level), occupation, skills and time.

At the beginning of the project, the SEEMP consortium decided to constraint the scope of the SEEMP ontology network to the IT domain, taking into account the resources available in the project and the validity of the results that would be reached with that scope. In addition, given that it was planned to use WSMO technology for data mediation, the project relied on WSML as the ontology implementation language.

\subsubsection{Reference ontology network architecture}

The decomposition into the areas mentioned above was crucial because it determined the ulterior decomposition of the reference ontology network into single or modular ontologies. Thanks to this decomposition, we identified, as parts or modules of the RO, three types of single ontologies that had to be built in the remaining development process (see Fig. 5): application ontologies (job offer and job seeker), domain ontologies (such as occupation, economic activity, etc.), and general ontologies (time). This way of classifying the ontologies can be found in Gómez-Pérez, Fernández-López, and Corcho (2003).

This classification was especially relevant since each of these types required a totally different development process, as can be seen next:

- Application ontologies were built from scratch within Scenario 1, taking into account the HR-XML recommendations on how the structure of a CV or a JO must be.

- Domain ontologies were built by applying Scenario 2, as a result of reusing and re-engineering non-ontological resources (NOR), such as the available international standards ${ }^{12}$ (NACE, ISCO-88 (COM), FOET, SOC,${ }^{13}$ ISIC, ${ }^{14}$ NAICS,${ }^{15}$ ISCED97, Pacific Exchange, ${ }^{16}$ CEFR,${ }^{17}$ Regions of the World, ${ }^{18}$ EU Driving License ${ }^{19}$ ), the ES proprietary classifications and the international codes (like ISO $3166,{ }^{20}$ ISO $6392,{ }^{21}$ ISO $4217^{22}$ ) that best fitted the ontology requirements. Fig.6 illustrates the knowledge-aware resources used for building each particular ontology.

- General ontologies were built by applying Scenario 4, as a result of reusing and re-engineering ontological resources as a whole.

Once domain and general ontologies were built and implemented, they were integrated with Application ontologies within

\footnotetext{
${ }^{11}$ Competency questions (CQs) (Grüninger \& Fox, 1994) were defined as questions that the ontology to be built should be able to answer.

12 The standards used in the RO network building can be downloaded from http:// ec.europa.eu/eurostat/ramon/.

${ }^{13}$ http://www.bls.gov/soc/.

14 http://unstats.un.org/unsd/cr/registry/regcst.asp?Cl=17\&Lg=1.

15 http://www.census.gov/epcd/www/naics.html.

16 http://fx.sauder.ubc.ca/currency_table.html.

17 http://www.coe.int/t/dg4/linguistic/CADRE_EN.asp.

18 http://park.org/Regions/.

19 http://ec.europa.eu/transport/road_safety/behavior/driving_licence_en.htm.

20 http://www.iso.org/iso/english_country_names_and_code_elements.

$21 \mathrm{http}: / /$ www.iso.org/iso/iso_catalogue/catalogue_tc/catalogue_detail.htm? csnumber $=4767$.

${ }^{22}$ http://www.iso.org/iso/en/prods-services/popstds/currencycodeslist.html.
} 


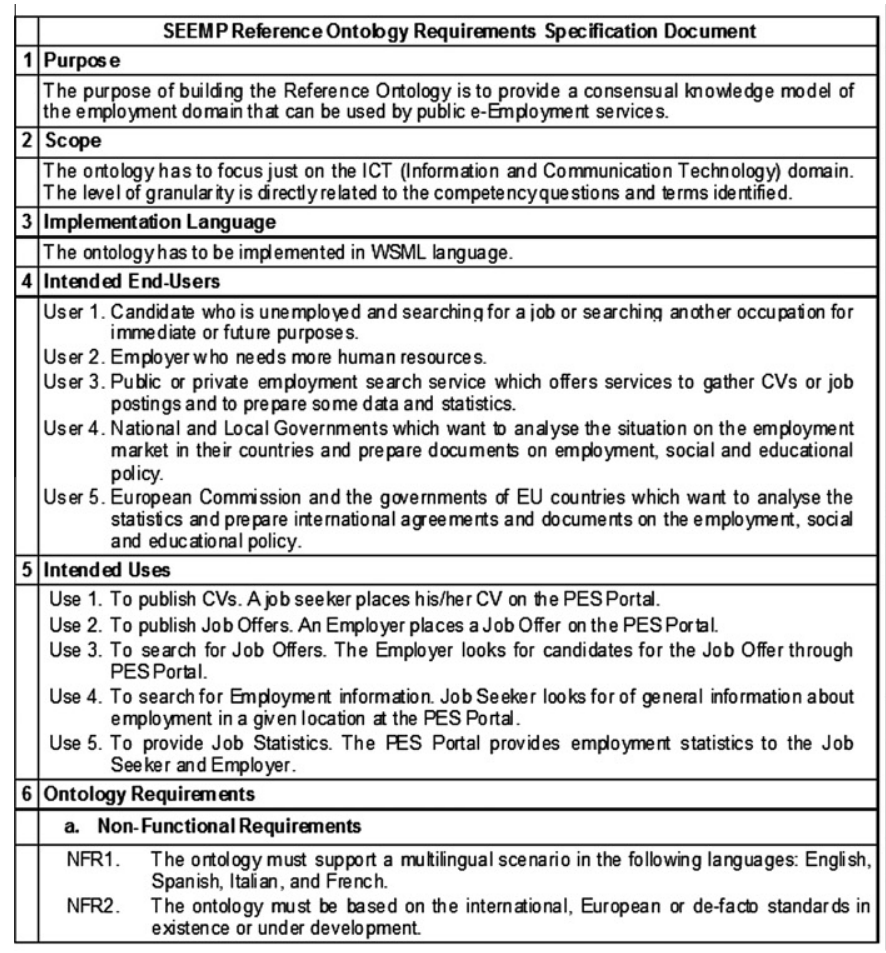

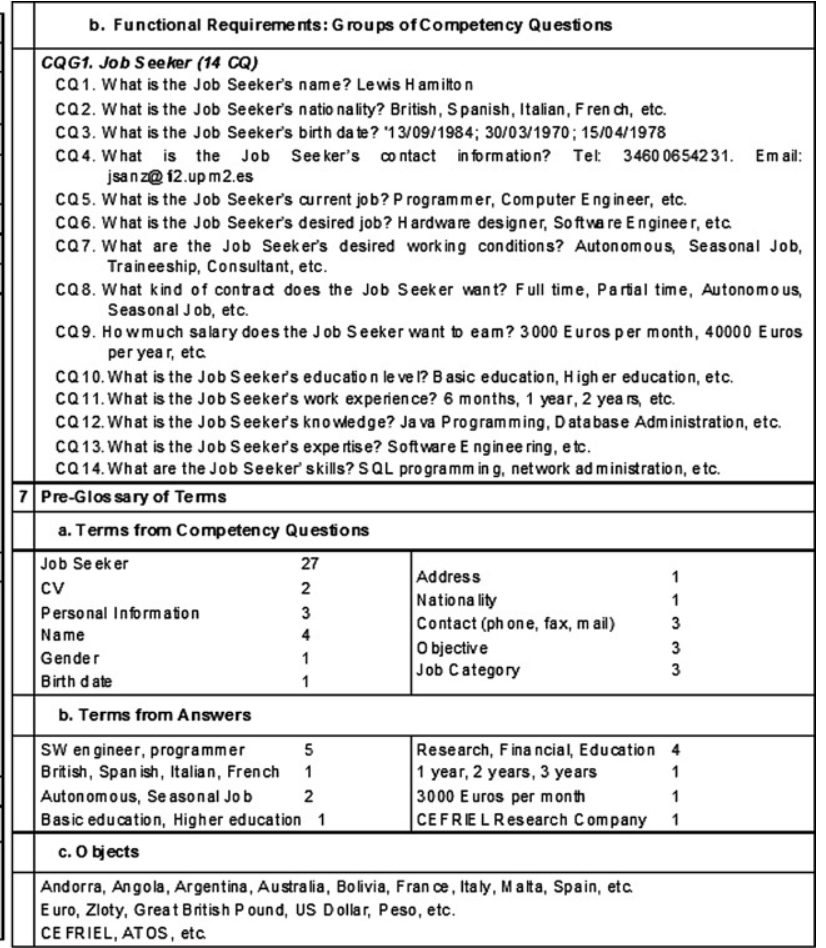

Fig. 4. ORSD

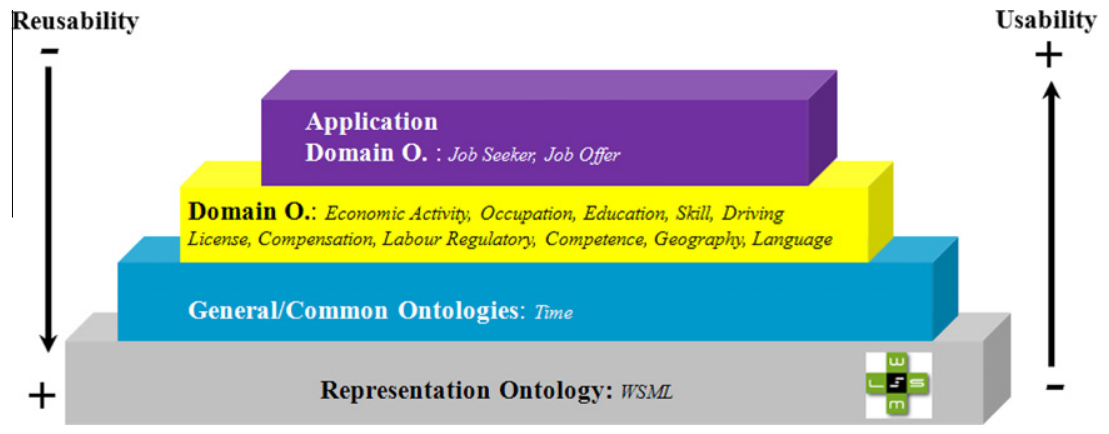

Fig. 5. Pyramid of ontology types.

Scenario 1 . As a result of this integration, we obtained the RO network. As can be seen in Fig. 6, the RO network comprises thirteen modular single ontologies: two application ontologies (Job offer and Job seeker) and ten domain ontologies, and one general ontology (Time). It is worth commenting here that thanks to the modular structure of the RO network (and of the local ontology networks, as can be seen in Section 5.2), the impact that the future changes in one single ontology may have on the rest of the single ontologies will be minimized. For example, replacing the occupation taxonomy with another taxonomy will only affect the occupation ontology (and the related semantic mappings defined with the local ontology networks).

Next, we provide a detailed explanation of the development process of each type of ontology.

\subsubsection{Development of application ontologies}

The main aim of the job seeker and job offer ontologies is to represent the structure of a CV and a JO respectively, which is depicted in Fig. 7. That is, these ontologies must specify the different constituting blocks or parts of a CV and a JO, and the information fields to be included in each block. For example, the job seeker ontology must define a $C V$ concept and include some attributes ${ }^{23}$ related to personal information, among them, an attribute has_nacionality_from of type Country, where Country is an imported concept from another single ontology called Geography. This Geography ontology is a domain ontology that defines all the different instances of the Country concept.

As was mentioned before, we defined the structure of a $\mathrm{CV}$ and a JO keeping in mind some HR-XML recommendations, and all the data models of the DB used by the ES involved. Regarding the job seeker ontology, the ontology defines a top level concept called Job_Seeker, which contains attributes for personal information and an attribute of type Candidacy. In turn, the Candidacy concept groups together information related to competences, spoken languages, education history, work experience and desired work conditions. Fig. 7 depicts the main concepts and ad hoc relationships among them that are defined in the job seeker ontology.

${ }^{23}$ Given the ontology language is WSML, we adopt its terminology and therefore we will use the term attribute instead of property, and type instead of range. 


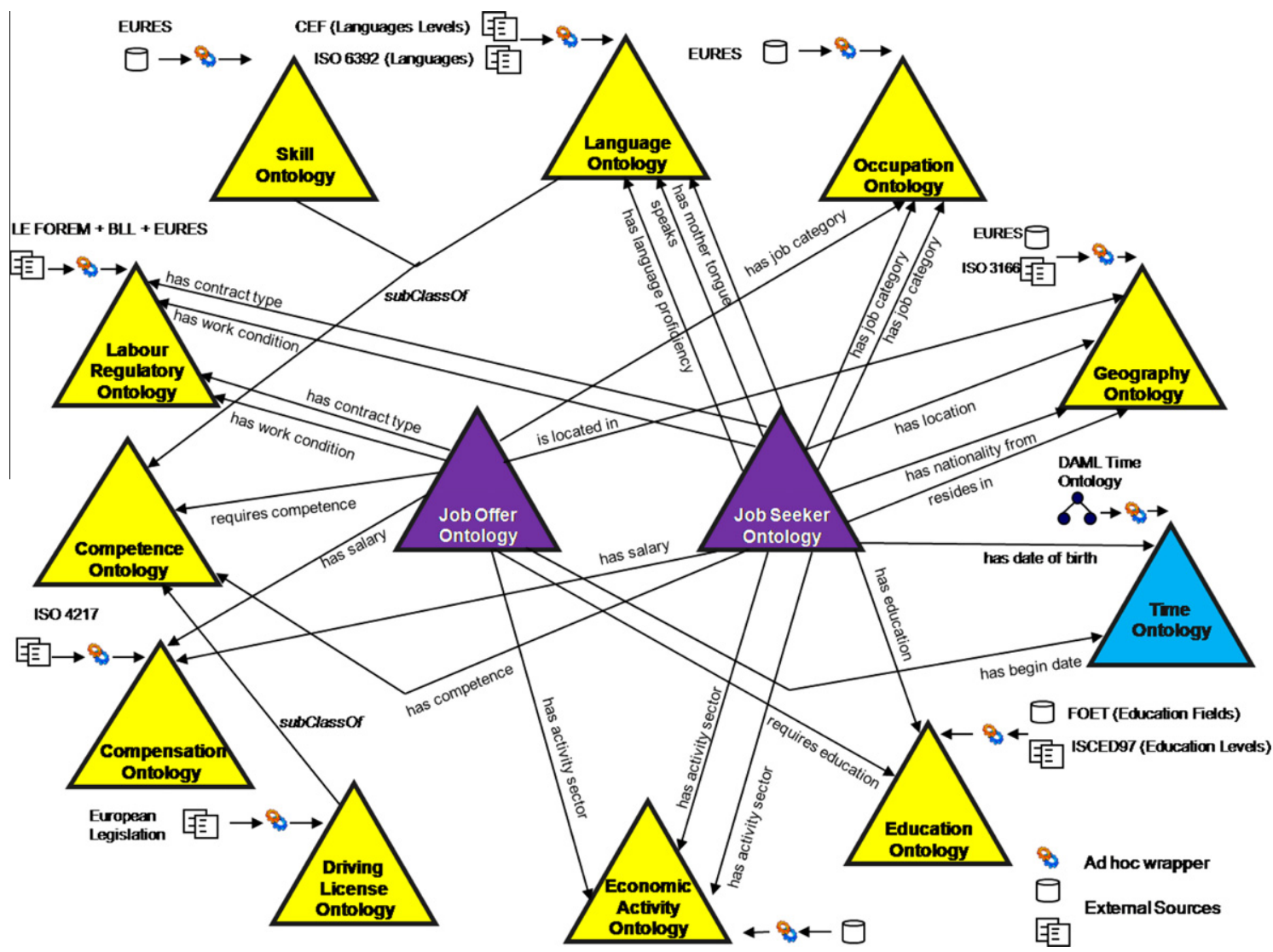

Fig. 6. SEEMP reference ontology network.

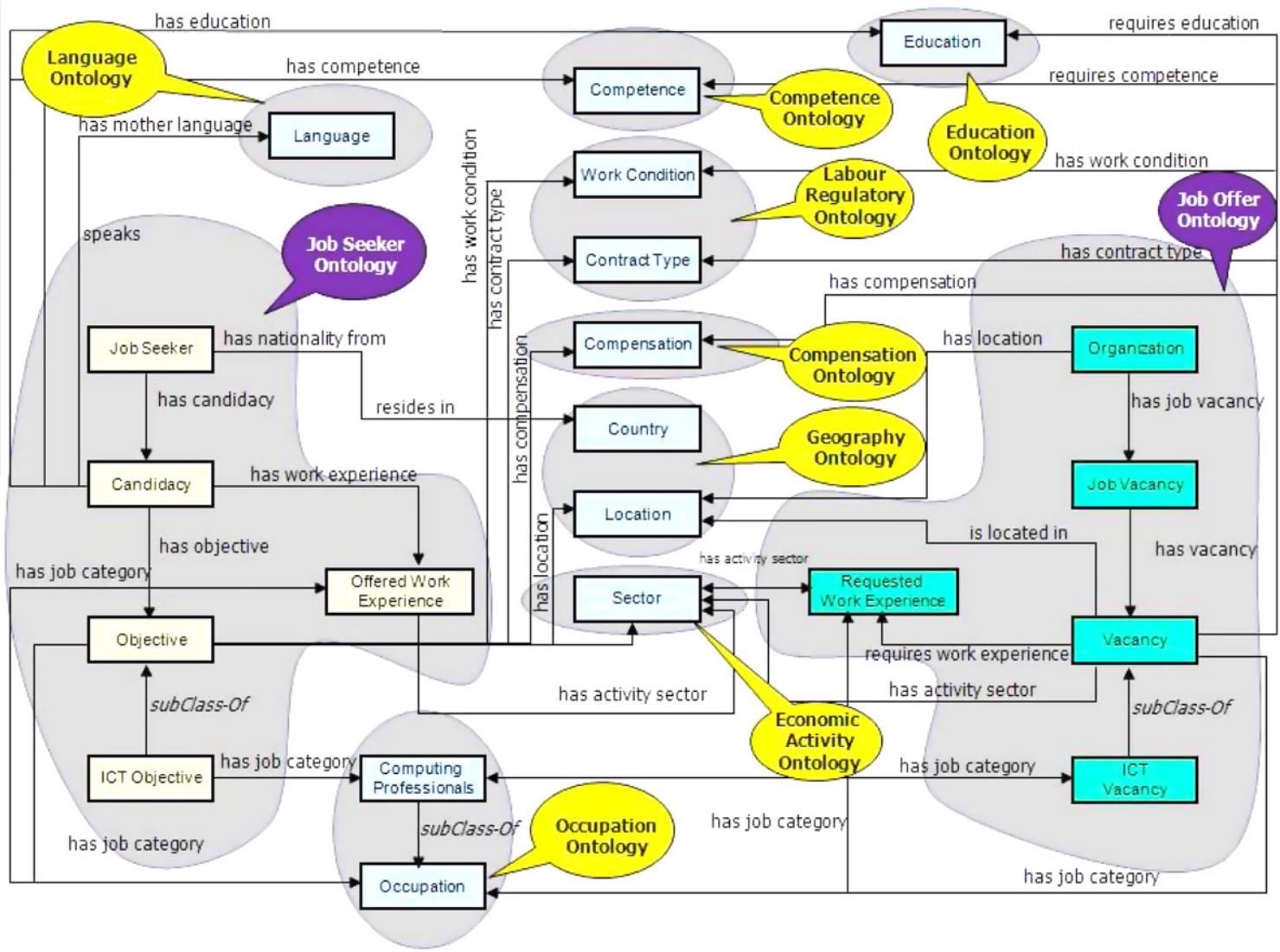

Fig. 7. SEEMP reference ontology - main concepts and relationships. 
Table 1

Standards, codes and classifications reused.

\begin{tabular}{|c|c|c|c|}
\hline Domain & Candidate standards/classifications & Selected standards/classifications & Justification \\
\hline Occupation & SOC, ISCO-88, ISCO-88 (COM), Eures classification & Eures classification & Best Coverage and European scope \\
\hline Economic sector & ISIC, NACE, NAICS & NACE & Best Coverage and European scope \\
\hline Education fields & ISCED 97, FOET & FOET & Best Coverage and European Scope \\
\hline Education levels & ISCED 97 & ISCED 97 & Worldwide scope, widely accepted \\
\hline Currency & Pacific exchange, ISO 4217, WordAtlas & ISO 4217 & Worldwide scope, widely accepted \\
\hline Geographic & ISO 3166, Regions of the World & ISO 3166 & Worldwide scope, widely accepted \\
\hline Language & ISO 639 & ISO 639 & Worldwide scope, widely accepted \\
\hline Language levels & CEFR & CEFR & European scope, widely accepted \\
\hline Driving license & EU Driving License & EU Driving License & European legislation \\
\hline Skills & Eures classification & Eures classification & Coverage and European scope \\
\hline Contract types & LE FOREM proprietary classification, ARL proprietary classification & Mix of both classifications & Acceptable Coverage in SEEMP scope \\
\hline Work condition & LE FOREM proprietary classification & LE FOREM proprietary classification & Acceptable Coverage in SEEMP scope \\
\hline
\end{tabular}

The figure also represents the imported concepts referenced in this ontology and taken from the domain ontologies.

Concerning the job offer ontology, this ontology defines a top level concept called Job_Vacancy, which contains information related to the employer, and an attribute of type Vacancy, which describes the occupation type, the job location, the work condition and contract type offered, and the conditions required to the applicant, such as competences (spoken languages, IT skills, etc.), work experience, and educational background. The main elements of this ontology appear in Fig. 7.

Thanks to the expressivity of WSML, some axioms in a datalog style were included in these ontologies. Most of them were used to define inverse relationships, and a few integrity constraints. Protocols for dealing with inconsistent mediated CVs or JOs have not been defined in the final version of the SEEMP prototype since this issue was considered beyond the project objectives. However, the existence of those integrity constraints may permit that kind of checking in future versions of the interoperability platform. A possible protocol for dealing with inconsistent information may be to label the information so that the final user (a job seeker or an employer) may be aware of it.

\subsubsection{Domain ontologies development}

The development of the domain ontologies has relied on reusing international standards and codes whenever possible. In some knowledge areas such as geography, currency, education levels, language, language levels and driving license, there is a unique and widely accepted worldwide/European standard; therefore, we only had to discover their existence and re-engineered them. In other areas, however, as in that of the occupation and economic sector, more than one candidate standard or NOR were available; consequently, a selection had to be carried out according to the following criteria: precision, coverage and consensus. The meaning of each of these criteria will be explained later on when the selection of the NOR for occupation is explained.

On the other hand, it also occurred that these standards did not exist in a worldwide or European scope, or that their adoption did not represent the best option from the perspective of the data mediation because some proprietary classification (or a mix of several ones) might offer lesser loss of information in the data mediation. That is the case of the occupation ontology, derived from the EURES proprietary classification and the labour regulatory ontology, whose contract types were taken from different sources (ARL proprietary classification and Le Forem proprietary classification). It is worth explaining that the selection of NORs for contract types and work condition was very pragmatic. This means that, since no international standards were available, we adopted an acceptable solution in the scope of the SEEMP project, in the sense of it provides an acceptable loss of information in the data mediation.
Table 2

Assessment table for occupation standard/classification candidates.

\begin{tabular}{llll}
\hline Non-ontological resources & Precision & Coverage & Consensus \\
\hline SOC & 0.0002 & $6 / \mathrm{K}$ & no \\
ISCO-88 & 0.0165 & $9 / \mathrm{K}$ & no \\
ISCO-88 COM & 0.0173 & $9 / \mathrm{K}$ & yes \\
ONET & 0.0179 & $21 / \mathrm{K}$ & no \\
EURES & 0.2507 & $89 / \mathrm{K}$ & yes \\
\hline
\end{tabular}

In any selection of NORs, the final result was assessed by domain experts.

We provide a table (see Table 1 ) that summarizes the selection of standards, codes, and classification accomplished for building every domain ontology.

In order to illustrate the process carried out to obtain a domain ontology following the methodological guidelines specified in Scenario 2, we are going to explain the building process of the occupation ontology. According to Scenario 2, a first set of NOR candidates (SOC, ISCO-88, ISCO-88 COM, ONET and EURES) was selected following the suggestions of the domain experts, and the precision, coverage and consensus of the NOR candidates were determined (see Table 2).

In order to calculate the precision and the coverage, the following formulas were used:

$$
\begin{aligned}
\text { Precision } & =\frac{\operatorname{card}\{\{\text { NORLexicalEntries }\} \cap\{\text { ORSDTerminology }\}\}}{\text { card }\{\text { NORLexicalEntries }\}} \\
& =\frac{\text { card }\{\text { NORLexicalEntriesITDomain }\}}{\text { card }\{\text { NORLexicalEntries }\}}
\end{aligned}
$$

$$
\begin{aligned}
\text { Coverage } & =\frac{\operatorname{card}\{\{\text { NORLexicalEntries }\} \cap\{\text { ORSDTerminology }\}\}}{\text { card }\{\text { ORSDTerminology }\}} \\
& =\frac{\operatorname{card}\{\text { NORLexicalEntriesITDomain }\}}{\operatorname{card}\{\text { ORSDTerminology }\}}
\end{aligned}
$$

where NORLexicalEntries is the set of all the terms defined in the NOR, NORLexicalEntries IT Domain is the set of all the IT occupations defined in the NOR, and ORSDTerminology is the set of all the possible IT occupations.

Given that all the possible IT domain occupations of the universe are not mentioned explicitly in the ORSD, and that it has been stipulated that the IT domain is the scope for the occupations to be represented in the occupation ontology, a constant $K$ is used in the calculations of the coverage that represents the cardinality of ORSDTerminology.

It was important for the project that the resources to be reengineered were focused on the current European human resource management because the user partners involved were European and because the out-coming prototype would be validated in 
European scenarios. Thus, domain experts established whether each candidate resource could count on the consensus of the European community in the human resource domain or not. They stipulated that only ISCO-88(COM) and EURES proprietary occupation classification may rely on the consensus of the European community.

After comparing these candidates, the chosen NOR was EURES; then, EURES went through a re-engineering process in order to obtain an ontological representation of itself. Within this activity we collected documentation about the EURES occupation classification from the company European Dynamics. From this documentation we found out that the EURES occupation classification is stored in two tables of a MS Access database following the snowflake data model (Malinowski \& Zimanyi, 2006). Then, we looked for a suitable re-engineering pattern for re-engineering NORs (Villazón-Terrazas et al., 2010) to transform a classification scheme represented as a snowflake data model into a taxonomy expressed at a conceptual level.

Because of the number of occupations of the EURES classification, it was not practical to create the ontology manually. Therefore, we created a software library, $\mathrm{NOR}_{2} \mathrm{O}^{24}$ (Villazón-Terrazas, Gómez-Pérez, \& Calbimonte, 2010), that read the data from the resource implementation and creates the corresponding classes and relations of the new ontology (already in WSML-flight) following the steps specified by the pattern chosen for re-engineering the EURES resource.

Fig. 6 shows the names of the standards and codes reused and re-engineered for building each domain ontology.

\subsubsection{General ontologies development}

When building the RO network, the only general ontology reused was the time ontology. According to the methodological guidelines specified in Scenario 4, the first task was to search for the ontology to be reused. To select the most suitable time ontology, we considered an existing comparative study (FernándezLópez \& Gómez-Pérez, 2004). We also took into account the terms related to time (date, date interval, and duration) extracted from the competency questions in the ORSD. We then assessed the time ontologies by means of the competency questions, then we selected the DAML time ontology, ${ }^{25}$ and finally we translated an OWL version of such an ontology into WSML without any loss of information, using some export/import services of the WebODE tool (Vega, Corcho, Fernández-López, \& Gómez-Pérez, 2003). In the context of Scenario 4, this translation can be seen as reengineering at the implementation level.

Given that the original OWL version does not contain the time axioms proposed by the DAML time ontology authors, the WSML-flight version does not contain them either; however, this lack of time axioms did not entail any problem because these axioms were not necessary at the SEEMP scope. It is worth mentioning that the use of WSML as ontology language leaves open the possibility of incorporating most of these axioms to the RO in the future, since they may be expressed in WSML and be used as integrity constraints.

\subsection{Local ontology network development}

According to the architecture explained in Section 3, each ES that participates in the SEEMP marketplace must make explicit the semantics of its local data sources in the form of a local ontology network. This means that a local ontology network created for an ES must support the representation of the CVs and JOs that the ES exchanges through XML messages. Inside these XML messages

\footnotetext{
24 http://www.oeg-upm.net/index.php/downloads/57-nor2o.

25 http://www.cs.rochester.edu/ferguson/daml/.
}

the ES includes codes related to the classifications (types of occupations, economic sectors, etc.) defined as part of its local data sources. Hence, also the local ontology network must somehow model these ES classifications. In this way, it will be possible to represent the CVs and JOs encoded in the XML messages by means of instances of the local ontology network.

Structurally, each local ontology network is very similar to the RO network, since both contain two single application ontologies (job seeker and job offer ontologies), which import terms from some domain ontologies (for representing types of occupation, economic sectors, etc.) and from a general ontology (the time ontology).

In order to build each local ontology network, the following three building approaches were considered:

- Building a local ontology network by adapting the RO network. The building process is structured/guided by the architecture of the RO network and the application needs. In this sense, we need to extend some elements already defined to remove unnecessary elements or to add the new application-dependent elements that appear in each ES schema source. The result of this should be a "structurally-similar RO" local ontology network. Thanks to this similarity, mappings between the local ontology network and the RO network should not result complex. On the other hand, mappings between the local ontology network and ES schema sources can be very complex.

- Building a local ontology network by reverse engineering the ES data sources. This process consists in applying a semi-automatic reverse engineering process to the data sources for obtaining some local ontological entities. In this case, mappings between the local ontology network and ES schema sources should not be complex. On the other hand, complex mappings will appear between the local and the RO network. This building process requires a very sophisticated engineering knowledge and greater familiarity with all the data and structures of the application.

- Building a local ontology network following a hybrid approach. This process consists in employing the most suitable approach of the two previous ones for each piece of the local ontology network to be built.

For the purpose of selecting an approach from the three commented above, we studied the XML schemas used by the different ESs involved in SEEMP for representing CVs and JOs. After that, we reached the conclusion that there were not significant differences among them in the sense that they considered more or less the same blocks of information (but with important syntactical differences). For example, if we take a CV of any of the ES, we will find information on personal data, education history, desired occupations, etc. Moreover, given that the RO network was built to model all the common information blocks used in CVs and JOs, it is very likely that most of these information blocks are somehow represented in terms of the RO network. On the other hand, important differences appeared when the classifications used by each ES for occupations, education titles, contract types, etc. were compared. Thus, except for the economic sector classification, where NACE standard is widely spread, and for the driving license classification, which is enforced by the European Commission, the other classifications were very heterogeneous. In order to build the local ontology network of the ES, in those specific cases in which the ES uses the same classification as the RO network, the domain subontology of the RO network that models this shared classification was directly taken from the RO network and reused without any reengineering process.

Taking into account these considerations, we selected the hybrid approach, building each subontology in this fashion: we built the application subontologies of the local ontology networks by reusing and re-engineering the application subontologies of the 


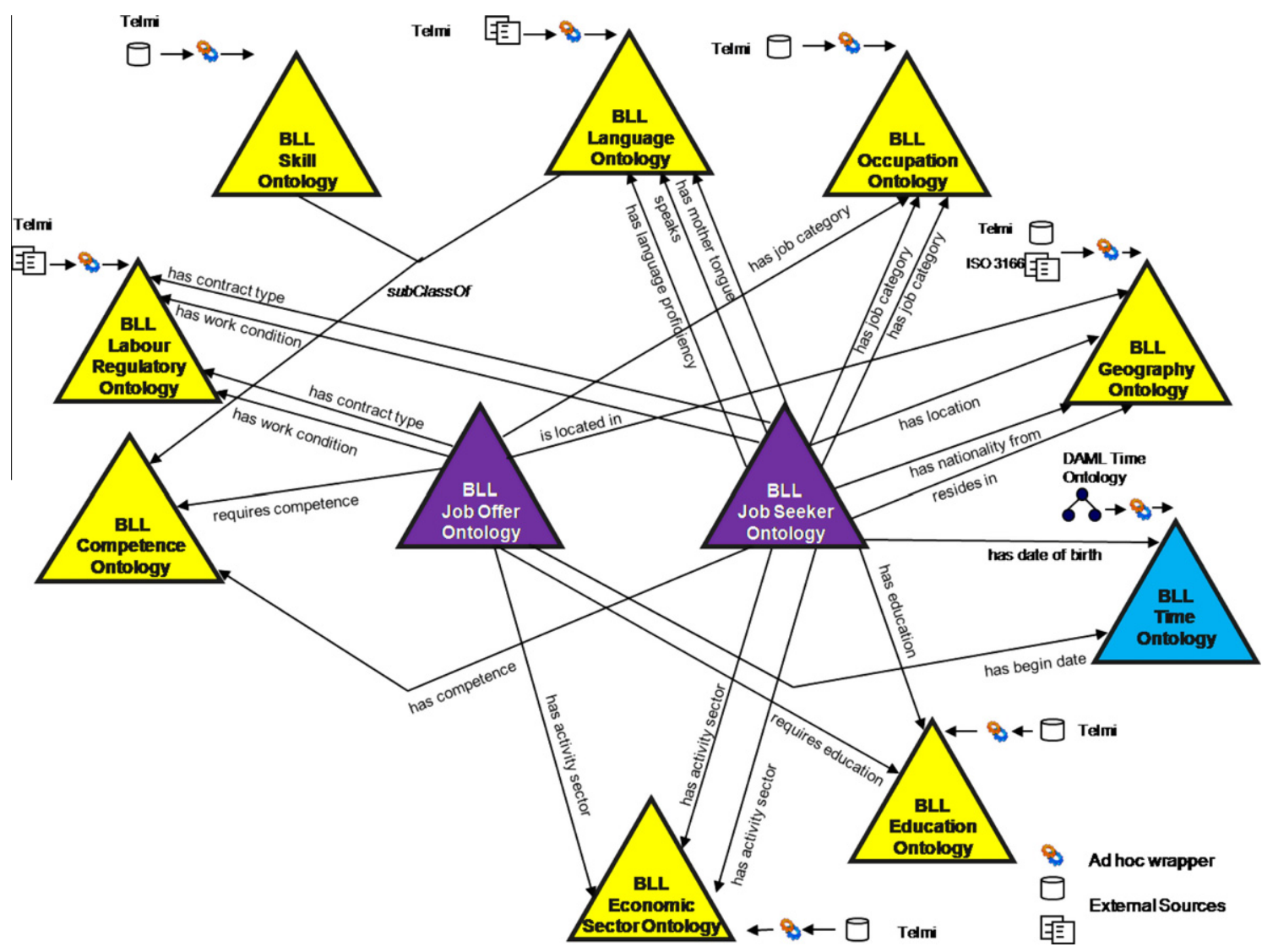

Fig. 8. Telmi ontology network.

RO network; and we built the domain subontologies of the local ontology networks as a re-engineering process from ES schema sources or, more simply, by reusing a domain subontology of the RO network.

As in the building of the RO network, a different approach was adopted to build each subontology type. Thus, Scenario 4 was used to build the application subontologies, whereas Scenario 2 was used to build the domain subontologies. The resulting ontologies were integrated in the context of Scenario 1.

\subsubsection{Development of application ontologies}

According to Scenario 4 presented in Section 4, after selecting the ontologies to be reused and re-engineered (application subontologies of the RO network), the following three activities had to be accomplished as part of the re-engineering process:

1. Ontological resource reverse engineering. This activity was skipped because the specifications and conceptual models of the RO network were available.

2. Ontological resource restructuring. This activity was performed at the specification level by comparing the specifications of the RO network application subontologies and their counterparts in the local ontology network to be built. When comparing the specifications, we distinguished three cases:

(a) Necessary element: if an element in a RO network application ontology is also required in its counterpart of the local ontology network, then this element must also be included in it and sometimes with minor changes (for example, a change in the range/type of an attribute).

(b) Unnecessary element: if an element in a RO network application ontology is not required in its counterpart of the local ontology network, then this element should be removed from it.

(c) Knowledge gap: if there is some missing element in a RO network application ontology that is required in its counterpart in the local ontology network, then this element should be added to the its counterpart in the local ontology network.

3. Ontological resource forward engineering. The application ontologies of the local ontology network were built from their counterparts in the RO network, taking into account the necessary and unnecessary elements and knowledge gaps identified at the specification level in the previous activity.

\subsubsection{Development of domain ontologies}

When it was not possible to reuse a domain ontology of the RO network because the ES used a different classification than the one modelled in the RO network for the same type of information, then it was necessary to apply Scenario 2 . In this case, the search and selection activities were skipped because the NORs to be reused were imposed by each ES. The remaining activities of this scenario were performed similarly as they were when the domain ontologies of the RO network were built (see Section 5.1.4).

The domain ontologies to be included in each local ontology network were chosen taking into account the features of the ES data sources to be modelled. More specifically, in the case of the two local ontology networks built within the SEEMP project, EURES and Telmi, both required domain ontologies for the same type of information as the RO network except for Telmi, which does not have a Compensation ontology (information related to currencies, job benefits as, for example, medical insurance, and salary period); this is so because this kind of information is not managed 
Table 3

SEEMP ontologies content statistics.

\begin{tabular}{llllll}
\hline Ontology & Concepts & Attributes & Axioms & Instances & $\begin{array}{l}\text { Efforts } \\
\text { (man-months) }\end{array}$ \\
\hline RO & 1985 & 315 & 1037 & 1449 & 6 \\
Telmi Local & 762 & 158 & 395 & 66 & 0.75 \\
EURES Local & 2577 & 240 & 1322 & 1433 & 0.6 \\
\hline
\end{tabular}

by Telmi ES. In Fig. 8 we can see the application, domain and general ontologies that constitute the Telmi ontology network.

\subsection{Creating the network of ontology networks}

In the previous sections we have explained how the RO network and the local ontology networks are built. However, there is a last step pending to obtain the whole network of ontology networks outlined in Section 3. The step consists in defining some alignments between each local ontology network and the RO network. A description of the approach followed for designing these alignments or semantic mappings can be found in Mocan, Cimpian, and Kerrigan (2006) and in SEEMP Consortium (2008). Basically, the development of these mappings consists of four steps that must be carried out in an iterative manner, and these are the following:

1. Specification of some test cases. Some test cases are specified according to the advice of the domain experts, who ensure the breath coverage of the XML schemas.

2. Specification of taxonomy mappings. The mapping development team must specify some code tables. A code table defines acceptable semantic equivalences between terms of a source ontology taxonomy and terms of the target ontology taxonomies.

3. Design of data transformations. The mapping development team must design some data transformations. A data transformation should specify how to compute a value from a set of values taken from instances of the source ontology and which value should be stored in an instance of the target ontology.

4. Implementation of mappings rules. Test cases, code tables and data transformations are implemented in the form of mapping rules and expressed in the Abstract Mapping Language syntax by means of the Web Service Modelling Toolkit. ${ }^{26}$

Every time a new ES wants to join the SEEMP marketplace, a new local ontology network should be built; additionally, it would be necessary to define the required alignments between this new local ontology network and the already existing RO network. From our experience in the development, we estimate that to incorporate a new ES to the SEEMP marketplace, i.e., developing a specific connector for the ES, would require around 6.35 man-months ( $\mathrm{MM})$, where $0.85 \mathrm{MM}$ would be for the construction of the local ontology network, and 0.7 MM would be for the development of the semantic mappings. The remaining 4.8 MM would be dedicated mainly to the development of (1) syntactical mappings between the XML schemas and the local ontology network; and (2) the Web Services infrastructure needed for enabling the communication between the ES connector and the EMP on the one side, and the connector and the ES on the other side.

\section{Conclusions}

This paper presents the development of the network of ontology networks that enables data mediation between ESs participating in

\footnotetext{
${ }^{26}$ http://sourceforge.net/projects/wsmt
}

an e-employment advanced system. This system relies on a semantic interoperability platform for dealing with heterogeneous information both in form and language. The ontology-based solution here proposed to build this complex system uses a network of ontology networks that combines a RO network and several local ontology networks. The network of ontology networks provides the semantics that enables the data mediation. Following this approach, the SEEMP consortium implemented a prototype, formed by the interoperability platform and three fully integrated ESs, capable of dealing with thousands of real CVs and JOs in the IT domain. At the end of the project, this prototype and the ontologies were evaluated (SEEMP Consortium, 2008) by the user partners with satisfactory results, and thus the technical approach adopted was validated.

Regarding the ontology construction, our experience in SEEMP has served us to demonstrate that the approach to building ontologies by reusing and re-engineering existing and already agreed upon non-ontological resources permits to build ontologies faster and with less resources; moreover, as a result of reusing resources, the ontologies can reach an immediate consensus and become reference ontologies in their respective domains.

Unlike previous e-employment ontologies that were built to be exploited in a regional or a national scope, the RO network here proposed was designed to support interoperability in a European scope. In addition, we must highlight that the RO network is publicly available and provides a richer vocabulary for representing JOs and CVs in the IT domain than previous e-employment ontologies. The expressiveness of the RO network was considered a critical issue in SEEMP, since the greater the expressiveness, the lower loss of information in the mediation process.

With respect to the application of the NeOn Methodology, we think that this methodology was especially useful for guiding the steps of the ontological engineers involved since it provides detailed and sufficient guidelines. In fact, we think that this methodology will be really valuable for guiding junior engineers who lack previous experience in building an ontology network, specifically if the network needs to be solidly grounded in NORs. In addition, we think that the existence of a well-defined and structured process for building the ontology network eases the planning, coordination and communication with other non Semantic Web members of the development team, which in turn helps to convey confidence in the final result.

It is also worth highlighting that the reuse scenarios posed by the NeOn Methodology has been put in practice for building a really huge network of ontology networks. This, in our opinion, strengthens the validity of our conclusions about the applicability of the NeOn Methodology. In order to illustrate the dimension of the ontologies and the effort performed by the ontological engineers for building them, we outline some data in Tables 3 and 4.

The size of these ontologies deserve a clarification. Most of the concepts, instances and axioms have been generated automatically by using wrappers, as was explained in Section 5 . With respect to the data related to effort, it must be said that they include, for each ontology, the effort related to the whole life cycle, i.e., from the ontology requirements specification activity to the implementation activity. In particular, in the case of the RO network effort, it must be added that at least $2 \mathrm{MM}$ were dedicated to the ontology

Table 4

SEEMP ontologies effort (man-months).

\begin{tabular}{llll}
\hline Ontology type & RO & Telmi Local & EURES Local \\
\hline Domain ontologies & 4 & 0.5 & 0.4 \\
Application ontologies & 2 & 0.25 & 0.2 \\
Total & 6 & 0.75 & 0.6 \\
\hline
\end{tabular}


specification activity (including the most intensive phase of the knowledge acquisition). In our opinion, a good ORSD helps to reduce the effort in the remaining activities of the ontology development; therefore, it is essential to dedicate a significant amount of time to this activity. If we compare the effort required, on one hand, by the application ontologies, and on the other hand, by the domain ontologies, we can observe a significant difference. Basically, the reason of this difference is that there are in the ontologies between three and seven different domain ontologies (occupation, economic activity, education levels, etc.), whilst there are just two application ontologies (job seeker and job offer ontologies). Thus, although an application ontology requires more effort than a domain ontology, the largest number of domain ontologies justifies that difference.

In addition, it is remarkable the difference between the effort for building the RO network (6 MM), and the effort for building the local ontology networks (0.75 MM and 0.6 MM). This difference exists because (1) most of the knowledge acquisition on human resources management was performed when building the RO network; and (2) when building the local ontology networks, a significant part of the RO network was reused, not only at the implementation level in form of ontology terms existing in the RO network, but also at the design level because of the architecture of the local ontology networks is very similar to the architecture of the RO network. Another important conclusion drawn from our experience is that it would have been impossible to achieve such low effort without the NeOn Methodology scenarios for reusing. These scenarios provided us some clear and detailed guidelines of the activities to carry out in each phase of the ontology network construction.

At this point, it must be pointed out that no other work related to the construction of an e-employment ontology has provided as detailed guidelines on the development process as we have done in this paper.

\subsection{Limitations of the proposed approach}

The limitations of our approach are mainly related to three aspects of the non-ontological resources: their availability, completeness and size. We are going to analyse the implications of each of them in the development of the RO network.

Regarding availability, as we have explained previously, the availability of standards and classifications have been crucial for the development of the RO network but what happens if we need to create a certain domain subontology, and it does not exist any international standard in that domain to be used as a reference? That would be a drawback for the project, which could be tackled by adopting a pragmatic solution: building the ontology from scratch and using resources during the knowledge acquisition, as we did with the domain of the contract types.

Regarding completeness, let us now suppose that this standard exists, but that it is not sufficiently complete, as it occurred when dealing with ISCO-88 COM. In that case, the resulting subontology derived from this standard will have to be enriched properly with the indispensable help of some domain experts; however, this process will increase the cost of building the RO network. In our case, that effort had already been carried out in a previous project that provided the EURES classification.

Finally, regarding size, let us suppose again that we have managed to build a good subontology for the target domain. However, this subontology is huge because the related standard is huge, as it occurred when tackling NACE. Above all, this should be a problem for the data mediation component, whose performance will be affected drastically since it relies on an inference engine whose computational time depends directly on the size of the ontology. When the SEEMP consortium implemented the prototype, we decided to use a manageable subset of NACE so that the performance of the data mediation component was acceptable. We expect that future advances in the development of more powerful inference engines help to alleviate this problem.

\section{Future work}

With the experience gained with the SEEMP project, we are able to enrich the NeOn Methodology, focusing on some issues that would be worth a further treatment. Among other things, this experience has shown us the importance of non-ontological resource quality, mainly because it will have a significant impact on the development costs. When we refer to non-ontological resource quality, we are referring to aspects such as that the resource is well documented and/or that it lacks anomalies (redundancies or inconsistencies)

Thus, in addition to a coverage analysis, a quality analysis should be carried out when selecting the non-ontological resources to be reused, as it is being done in the selection of the ontological resources.

Furthermore, the re-engineering of a non-ontological resource may be a good opportunity to improve the quality of this non-ontological resource, since the ontological version of a nonontological resource can be improved by means of state of art evaluation methods, possibly automatic, and then the ontological version may be transformed back to its non-ontological original format without, for example, inconsistencies.

An alternative line of study could be to analyse how to transform non-ontological resources that change over the time (like the standards and classifications that we used in SEEMP), to identify how the frequency of changes affects the ontologization of that resource, and to propose incremental transformations.

And finally, another interesting line of research may consist in studying to what extent the solution here proposed can be also applied in other domains such as e-health or open e-government, where a network of ontology networks may enable the required interoperability.

\section{Acknowledgements}

This work has been carried out under the SEEMP project (FP6-027347). We would like to thank the SEEMP partners for their support in the development of the ideas exposed in this paper. Without their suggestions and criticisms the SEEMP ontology network would have never become what it is now. Thanks to Rosario Plaza for her comments on the paper.

\section{References}

Biesalski, E., \& Abecker, A. (2005). Human resource management with ontologies. In Springer postproceedings: Workshop on IT tools for knowledge management systems: Applicability, usability, and benefits (KMTOOLS) (pp. 499-507).

Bizer, C., Heese, R., Mochol, M., Oldakowski, R., Tolksdorf, R., \& Eckstein, R. (2005) The impact of semantic web technologies on job recruitment processes. In International Conference Wirtschaftsinformatik (WI 2005), Bamberg, Germany.

Dorn, J., Naz, T., \& Pichlmair, M. (2007). Ontology development for human resource management. In: Stary, C., Barachini, F., Hawamdeh S. (Eds.), Proceedings of fourth international conference on knowledge management, Series on information \& knowledge management, (Vol. 6, pp. 109-120). Vortrag: fourth international conference on knowledge management, Wien; 2007-08-27 - 2007-08-28.

Espinoza, M., Montiel-Ponsoda, E., \& Gómez-Pérez, A. (2009). Ontology localization. In The fifth international conference on knowledge capture, KCAP 2009.

Fernández-López, M., \& Gómez-Pérez, A. (2004). Searching for a time ontology for semantic web applications. In: C. for Theoretical, A. Ontology (Eds.), Formal ontology in information systems, Lecture notes in computer science.

García-Sánchez, F., Martínez-Béjar, R., Contreras, L., Fernández-Breis, J. T., \& Castellanos-Nieves, D. (2006). An ontology-based intelligent system for recruitment. Expert Systems with Applications, 31(2), 248-263<http://www. sciencedirect.com/science/article/B6V03-4H80S9G-2/2/bb9b93d92f84f01fe 1 652edc7007c496>. 
Gómez-Pérez, A., Fernández-López, M., \& Corcho, O. (2003). Ontological engineering. Advanced information and knowledge processing. Verlag: Springer.

Grüninger, M., \& Fox, M. S. (1994). The role of competency questions in enterprise engineering. In IFIP WG5.7 Workshop on benchmarking - theory and practice.

Gualtieri, A., \& Ruffolo, M. (2005). An ontology-based framework for representing organizational knowledge. In International conference on knowledge management (I-KNOW 05), Graz.

Haase, P., Rudolph, S., Wang, Y., Brockmans, S., Palma, R., Euzenat, J., \& d'Aquin, M. (2006). Networked Ontology Model, Tech. rep., NeOn project deliverable D1.1.1

Malinowski, E., \& Zimanyi, E. (2006). Hierarchies in a multidimensional model: From conceptual modeling to logical representation. In Data and knowledge engineering.

Mocan, A., Cimpian, E., \& Kerrigan, M. (2006). Formal model for ontology mapping creation. In I. F. Cruz, S. Decker, D. Allemang, C. Preist, D. Schwabe, \& P. Mika, et al. (Eds.), International semantic web conference. Lecture notes in computer science (Vol. 4273, pp. 459-472). Springer.

Mochol, M., Paslaru, \& E. (2006). Bontas Simperl, Practical guidelines for building semantic erecruitment applications. In Proceedings of the internationa conference on knowledge management (iKnow'06). Special Track: Advanced Semantic Technologies.

Presutti, V., Daga, E., Gangemi, A., \& Blomqvist, E. (2009). Extreme design with content ontology design patterns. In Proceedings of the workshop on ontology patterns (WOP 2009), collocated with ISWC 2009, CEUR workshop proceedings, Vol. 516

SEEMP Consortium (2008). SEEMP Platform User Manual, Tech. rep., SEEMP project deliverable D5.6.

SEEMP Consortium (2008). Final Pilot Testing and Evaluation, Tech. rep., SEEMP project deliverable D6.3.

SEEMP Consortium, (2006).Supporting State of the Art, Tech. rep., SEEMP project deliverable D3.2
Suárez-Figueroa, M. C. (2010). NeOn Methodology for building ontology networks: Specification, scheduling and reuse. Ph.D. thesis, Universidad Politécnica de Madrid, Spain. URL <http://oa.upm.es/3879>.

Suárez-Figueroa, M. C., \& Gómez-Pérez, A. (2008). First attempt towards a standard glossary of ontology engineering terminology. In The 8th international conference on terminology and knowledge engineering, Managing ontologies and lexical resources. TKE 2008 (pp. 1-15).

Terziev, I., Kiryakov, A., \& Manov, D. (2005). Base upper-level ontology (BULO) Guidance, Tech. rep., SEKT project deliverable D1.8.1

Trichet, F., \& LeclFre, M. (2003). A framework for building competency-based systems dedicated to human resource management. In N. Zhong, Z. W. Ras, S. Tsumoto, \& E. Suzuki (Eds.), ISMIS. Lecture notes in computer science (vol. 2871, pp. 633-639). Springer.

Valle, E.D., Cerizza, D., Celino, I., Fugini, M., Estublier, J., Vega, G., Kerrigan, M. Gómez-PTrez, A., Ramírez, J., Villazón-Terrazas, B., Zhao, G., Cesarini, M., \& Paoli, F. D. (2008). The SEEMP approach to semantic interoperability for eemployment. In Premier reference source (pp. 105-126).

Vega, J. C. A., Corcho, Ó., Fernández-López, M., \& Gómez-Pérez, A. (2003). WEBODE in a nutshell. AI Magazine, 24(3), 37-48.

Villazón-Terrazas, B. (2011). A method for reusing and re-engineering nonontological resources for building ontologies. Ph.D. thesis, Universidad Politécnica de Madrid, Spain

Villazón-Terrazas, B., Gómez-Pérez, A., \& Calbimonte, J. P. (2010). NOR 2 O: A library for transforming non-ontological resources to ontologies. In Proceedings of the seventh extended semantic web conference (ESWC 2010).

Villazón-Terrazas, B. Suárez-Figueroa, M. C. \& Gómez-Pérez, A. (2010). A patternbased method for re-engineering non-ontological resources into ontologies. International Journal on Semantic Web and Information Systems, 6(4), 27-63.

Wache, H., Vgele, T., Visser, U., Stuckenschmidt, H., Schuster, G., Neumann, H. et al. (2001). Ontology-based integration of information - a survey of existing approaches, 108-117. 